\title{
Nanoformulation des corps gras. Nanocapsules, nanoémulsions
}

Jean-Thierry SIMONNET

Pascal RICHART

Odile AUBRUN

Florence L'ALLORET

L'Oréal Recherche,

188, rue Paul Hochart,

94152 Chevilly-Larue, France

$<$ flalloret@recherche.loreal.com>

Abstract: Nanocapsules (submicron particles, of diameter $250 \mathrm{~nm}$, with an oily core enclosed in a solid polymeric shell) were prepared using a controlled nanoprecipitation process with preformed polymers (for example poly- $\epsilon$ caprolactone, polyethylene adipate). Nanocapsules formed by this method contain up to $7.5 \%$ oil (1\% polymer). The following properties are observed using nanocapsules: improved oxygen stability of active ingredients (retinol, $\beta$ carotene), significantly improved vectorisation (up to 3 times) in skin and a reservoir effect. Due to their solid polymer shell nanocapsules are stable when formulated and penetrate intact into the superficial layers of the epidermis. Nanoemulsions wich are very fine oil-in-water dispersions having droplet diameter smaller than 100 nanometers, were prepared with a high shear device. Two major sources of unstability were identified and extensively studied: Ostwald ripening and depletion induced floculation following the addition of thickening polymers. The control of these two mechanisms allowed the industrial production of a large variety of cosmetic products. The nanoemulsions are easily valued in skin care due to their good sensorial properties and their biophysical properties.

Key words: nanoemulsions, Ostwald ripening, depletion, nanocapsules, nanoprecipitation, vectorisation

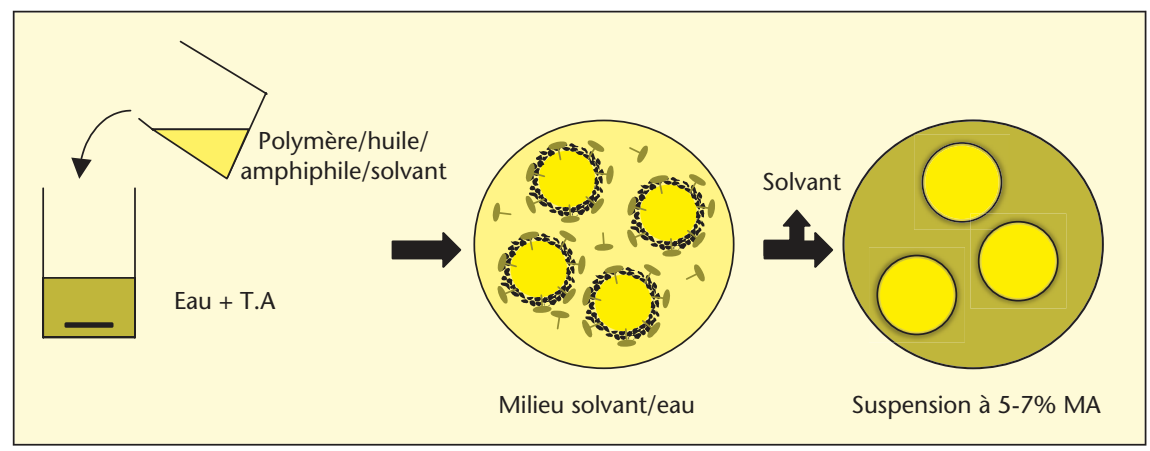

Figure 1. Procédé de fabrication des nanocapsules par émulsification spontanée et basculement de solvant.

ou lipophiles et leur vectorisation dans les structures cutanées.

\section{Principe d'émulsification et procédé}

II est possible de faire des capsules par de nombreux procédés mais rares sont ceux qui permettent de faire des capsules submicroniques. Récemment Tiarks et al. [1] ont proposé un procédé de formation de nanocapsules par polymérisation en miniémulsion. Ce procédé est dépendant d'un outil de dispersion très performant comme les ultrasons ou l'homogénéisation haute pression.

Nous utilisons un procédé basé sur l'émulsification spontanée d'une phase huileuse par son basculement via un solvant organique dans une phase aqueuse, sous agitation lente (barreau magnétique ou pâles) comme présenté à la figure 1.

L'un des mécanismes de l'émulsification spontanée est la croissance des gouttes dans des régions supersaturées qui s'amorcent lorsque le solvant transfert de la phase organique vers la phase aqueuse non encore équilibrée [2]. Par ailleurs, dans des systèmes contenant des tensio-actifs et sous l'effet de l'agitation, des variations de tensions interfaciales, dues à la formation d'un gradient de tensio-actif sous l'effet de turbulence générée par la diffusion du solvant (effet Marangoni), conduisent à la formation de gouttes stables de très faible taille [3].

Dans notre système, le polymère et le lipide amphiphile d'enrobage sont eux aussi solubilisés dans le solvant organique. Le solvant organique doit être parfaitement miscible à l'eau, pour permettre, lors de son introduction dans la phase aqueuse qui elle-même contient un tensio-actif, l'émulsification spontanée de la phase huileuse, la précipitation du polymère à la surface de la gouttelette formée et le dépôt de la phase lamellaire à la surface des particules. La théorie de formation de telles capsules a été décrite par Torza et al. en 1970 [4].

La formation et la taille des capsules obtenues vont dépendre de plusieurs paramètres :

- le solvant doit être parfaitement miscible à l'eau : acétone, éthanol, isopropanol ; 
- le polymère doit être soluble dans le solvant et pas dans la phase aqueuse ;

- la nature de l'huile. Son poids moléculaire doit être supérieur à $500 \mathrm{~g} / \mathrm{m}$ pour éviter tout phénomène de mûrissement d'Ostwald ;

- la nature de l'amphiphile. II doit former spontanément une phase cristal liquide de type lamellaire ;

- le ratio solvant/eau peut être compris entre 0,05 et 0,5 . Plus celui-ci est faible, plus petites sont les nanocapsules;

- le taux d'huile, de polymère et de lipide amphiphile dans le solvant organique doit être faible, moins de $15 \%$ /solvant ;

- les volumes mis en jeu représentent de 3 à 10 fois le volume final après évaporation du solvant organique et d'une grande quantité d'eau sous pression réduite.

Il est possible de contrôler la taille des capsules de 130 à $600 \mathrm{~nm}$.

Dans nos conditions : ratio solvant/eau de 0,5 , ratio huile/polymère de 5,5\% d'huile dans la suspension finale, $7 \%$ ( $d$ 'huile + polymère + lipide amphiphile) dans la phase organique et $0,5 \%$ final d'un poloxamer dans la phase aqueuse, les nanocapsules produites ont une taille de l'ordre de $250 \mathrm{~nm}$ avec une polydispersité très réduite. Ces capsules sont stables plusieurs années à température ambiante.

Ce procédé, bien que nécessitant des volumes de solvant et d'eau importants, a l'avantage d'assurer la formation des capsules dans des conditions de température $\left(20\right.$ à $\left.45^{\circ} \mathrm{C}\right)$ et d'agitation douces, particulièrement favorables à l'encapsulation de molécules sensibles comme les vitamines.

\section{Nanocapsules}

\section{Structure}

Les nanocapsules telles que décrites sont des particules constituées par un cœur huileux, enrobé par une coque polymérique, elle-même pouvant être enrobée par une phase lipidique de type lamellaire. L'ensemble a un diamètre inférieur au micron (figure 2).

Les molécules encapsulées doivent être soit huileuses soit solubles dans une huile dite support. La coque polymérique apporte une protection solide à la particule, lui permettant de ne pas être sensible au cisaillement lors de I'application sur la peau. Quant à l'enrobage lamellaire, il permet d'améliorer l'étanchéité de la particule vis-à-vis des composés huileux externes et de favoriser la pénétration de la particule dans les structures cutanées par analogie de structure avec les lipides intercornéocytaires, eux aussi sous forme lamellaire.

\section{Composition}

\section{Polymères}

Historiquement, Al Khouri et al. et Gallardo et al. [4-6], ont utilisé des monomères d'alkyl

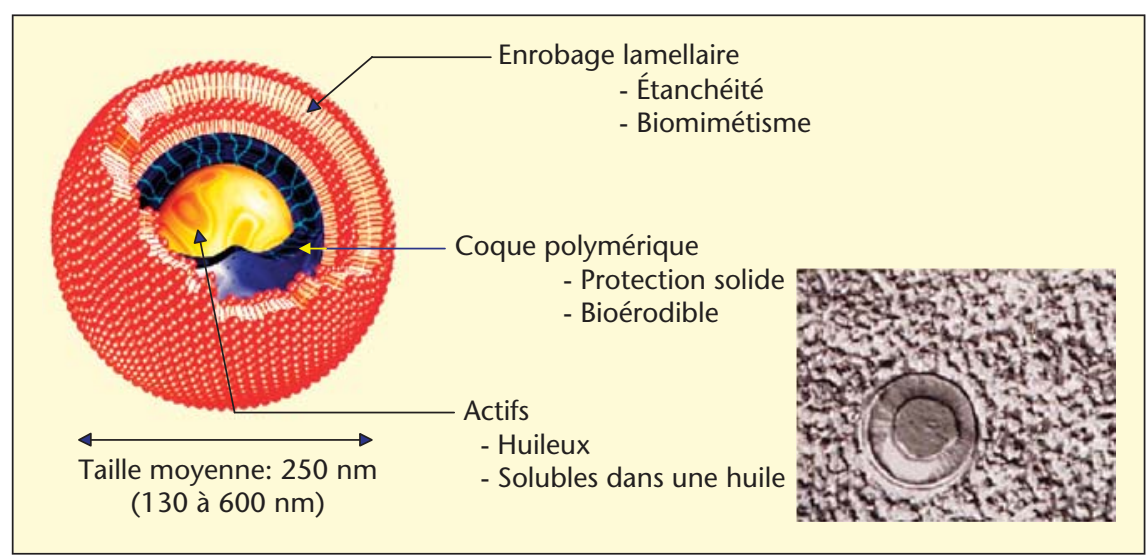

Figure 2. Structure des nanocapsules. cyanoacrylate (ethyl, butyl ou isobutyl) qui, au contact de l'eau, par polymérisation interfaciale, polymérisent spontanément et forment la paroi de la capsule. II est cependant préférable d'utiliser des polymères pour éviter des problèmes d'innocuité cutanée liés à la présence potentielle de monomères résiduels. La formation de la coque se faisant par nanoprécipitation du polymère autour du globule d'huile.

Les polymères envisageables peuvent être biodégradables ou non. Parmi les polymères les plus utilisés, on préfère choisir les dérivés de la cellulose tels que l'acétopropionate de cellulose, les polyéthylènes adipates, les poly- $\epsilon$ caprolactones, les polyesters sulfonés. Ceux-ci doivent être solubles dans le milieux organique de départ et insolubles dans I'eau [7-13].

\section{Enrobage lipidique}

Cet enrobage lipidique doit être sous la forme $d^{\prime}$ un cristal liquide lamellaire pour remplir totalement ses fonctions. La première est de parfaire l'étanchéité de la capsule lorsque celle-ci est dispersée dans un milieu de type émulsion $H / E$, la seconde est d'améliorer la pénétration des nanocapsules dans le stratum corneum pour qu'elles y jouent leur effet réservoir. Le choix des lipides doit se faire sur leur propriété à former spontanément, au contact de l'eau, une phase cristal liquide lamellaire.
L'amélioration de l'étanchéité des capsules est mesurée par la mise en émulsion d'une suspension de nanocapsules contenant de la vitamine $E$ avec de I'huile. La fraction huileuse est ensuite séparée par centrifugation et la vitamine $\mathrm{E}$ est dosée dans celle-ci. Les résultats (tableau 1) sont exprimés en pourcentage de fuite.

Dans tous les cas, on constate que l'enrobage lamellaire inhibe l'extraction de la vitamine $\mathrm{E}$ hors des capsules. Cette propriété est renforcée par les effets de stabilisation vis-à-vis de l'oxydation constatée sur certaines molécules. Les lipides concernés sont choisis parmi les lécithines de soja, les diméthicones copolyols, les alkylesters de polyols associés ou non à du cholestérol.

\section{Molécules encapsulées}

Les molécules encapsulées doivent être hydrophobes, liquides à température ambiante ou être solubles dans une huile dite support. A titre d'exemple, on peut citer les triglycérides caprique/caprylique, la vitamine E, l'acétate de vitamine $E$, certaines huiles végétales, l'octyl dodécanol, le palmitate de vitamine $A$, le rétinol, le $\beta$-carotène, le lycopène... Le taux de polymère est adapté de telle façon que le rendement d'encapsulation est de $100 \%$. Le taux final de molécule encapsulée est compris entre 2 et $7 \%$. Le ratio huile/polymère est quant à lui de l'ordre $5 / 2$ à $5 / 1$.
Tableau 1. Pourcentages de fuite des nanocapsules en comparaison avec une émulsion de même taille, pour différentes natures d'huile.

\begin{tabular}{|lcc|}
\hline Huile & $\begin{array}{c}\text { Témoin sans enrobage } \\
\mathbf{( \% )}\end{array}$ & $\begin{array}{c}\text { Enrobage lamellaire } \\
\text { (\%) }\end{array}$ \\
\hline Octyl dodécanol & 78 & 0 \\
Huile d'amande d'abricot (triglycérides) & 81 & 0 \\
Silicone volatile & 84 & 0 \\
Huile de macadamia (triglycérides) & 84 & 0 \\
Huile de pépins de raisin & 86 & 0 \\
Perhydrosqualène & 88 & 0 \\
\hline
\end{tabular}


Tableau 2. Pourcentages de protection contre le rayonnement UV après application de nanocapsules, en comparaison avec une émulsion de même taille.

\begin{tabular}{|ccc|}
\hline $\begin{array}{c}\text { Intensité de I'exposition } \\
\text { UV (MED) }\end{array}$ & $\begin{array}{c}\text { Traitement par } \\
\text { des nanocapsules } \\
(\%)\end{array}$ & $\begin{array}{c}\text { Traitement par } \\
\text { une émulsion H/E } \\
\text { (\%) }\end{array}$ \\
\hline 1,25 & 44 & 5 \\
0,75 & 81 & 44 \\
\hline
\end{tabular}

\section{Propriétés de nanocapsules}

\section{Potentialisation de l'activité} des molécules encapsulées

Effet de la vitamine $\mathrm{E}$ sur la protection du relief cutané après exposition UV à 0,75 et 1,25 MED (Minimal Erythemal Dose). La vitamine E est formulée dans des nanocapsules ou dans une émulsion $\mathrm{H} / \mathrm{E}$ au taux final de $1 \%$. Les applications cutanées sur 8 volontaires ont été réalisées quotidiennement pendant 4 jours avant I'exposition UV et pendant les 4 jours d'exposition aux UV. Les résultats (tableau 2) sont exprimés en pourcentage de protection calculé par rapport à une zone témoin n'ayant pas reçu de formulation à la vitamine $\mathrm{E}$.

Par ailleurs, un effet dose est vérifié, de 1 à $3 \%$ de vitamine $E$ sur l'érythème et le relief cutané. Sans ajout de filtre solaire, on constate un bon effet protecteur de la vitamine E vis-à-vis des UV lorsqu'elle est nanoencapsulée. Ce type de résultat est également constaté lors d'études cliniques faites sur d'autres actifs.

\section{Biodisponibilité cutanée \\ des molécules encapsulées}

Ces études sont réalisées sur peau humaine isolée, sur cellule de Franz. La pénétration comparative de la vitamine E radiomarquée, entre une suspension de nanocapsules (introduites dans une émulsion $H / E$ ) et une émulsion $H / E$, est quantifiée dans les structures cutanées à l'aide d'un compteur à scintillation après «slicing » de l'échantillon de peau. $2 \mathrm{mg} / \mathrm{cm}^{2}$ de produit sont appliqués pendant 16 heures. On observe un effet réservoir renforcé du stratum corneum et des nanocapsules, et une pénétration de la vitamine $\mathrm{E}$ dans l'épiderme vivant multiplié par trois avec les nanocapsules par rapport à l'émulsion témoin.

En microscopie confocale Raman, il est possible de suivre la cinétique de pénétration dans les structures cutanées du rétinol. Une étude comparative a été réalisée sur peau humaine isolée, entre une suspension de nanocapsules et une émulsion de taille identique $(250 \mathrm{~nm})$. Toutes deux contenants $0,5 \%$ de rétinol.

On constate (figure 3 ) que le rétinol nanoencapsulé est véhiculé efficacement dans l'épiderme vivant, là où son métabolisme est le plus important, lorsqu'il est nanoencapsulé. Formulé en émulsion, même si les globules huileux de celle-ci ont une taille comparable à celle des stratum corneum.

Par ailleurs, la cinétique de pénétration indique que c'est au bout de six heures après l'application que le rétinol atteint l'épiderme vivant (figure 4).

Enfin, parce que les nanocapsules sont de faible taille et solides, il est possible d'en trouver intactes sous les premières couches cellulaires nanocapsules, le rétinol reste au niveau du du stratum corneum (figure 5). II s'agit de la preuve que des particules de cette taille (250 $\mathrm{nm}$ ) peuvent pénétrer dans la peau.

Une suspension de nanocapsule a été appliquée sur cinq volontaires à peau sèche ou normale pendant 21 jours à raison d'une fois par jour. À $\mathbf{2 2}$, un prélèvement par strip (cornéodisque) a été réalisé, ayant pour effet $d$ 'arracher les premières couches du stratum corneum. Ces prélèvements sont ensuite observés en MEB (figure 5). Sur tous les prélèvements, on constate la présence de nanocapsules intactes.

\section{Stabilisation des molécules encapsulées}

Les molécules encapsulées sont biologiquement actives au niveau cutané et souvent sensibles à l'oxydation ou à l'hydrolyse. L'encapsulation de celles-ci dans des nanocapsules permet d'améliorer significativement leur sta-

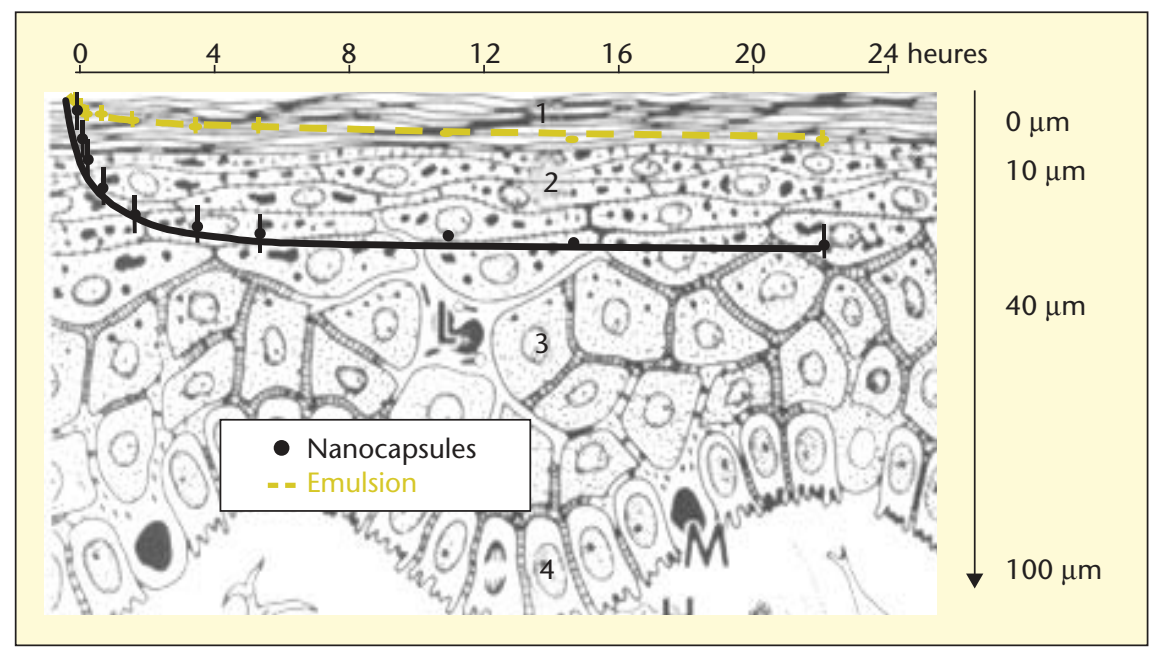

Figure 3. Cinétique de pénétration du rétinol dans les structures cutanées, suivie par microscopie confocale Raman.

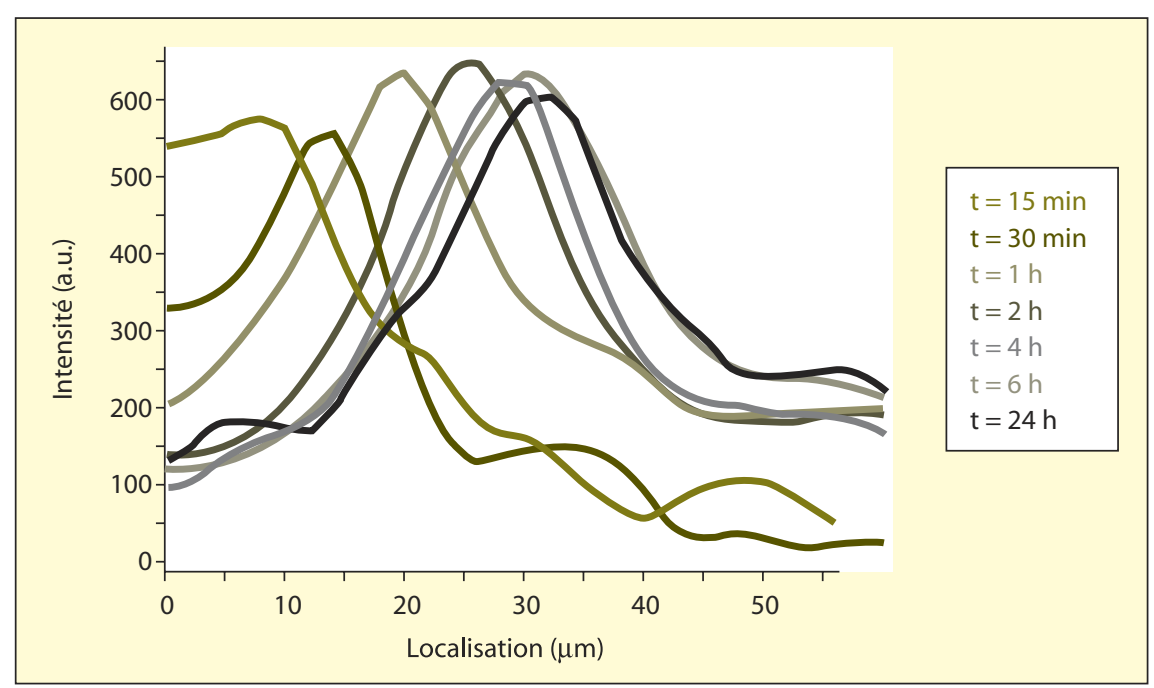

Figure 4. Localisation du rétinol dans les structures cutanées en fonction du temps. 


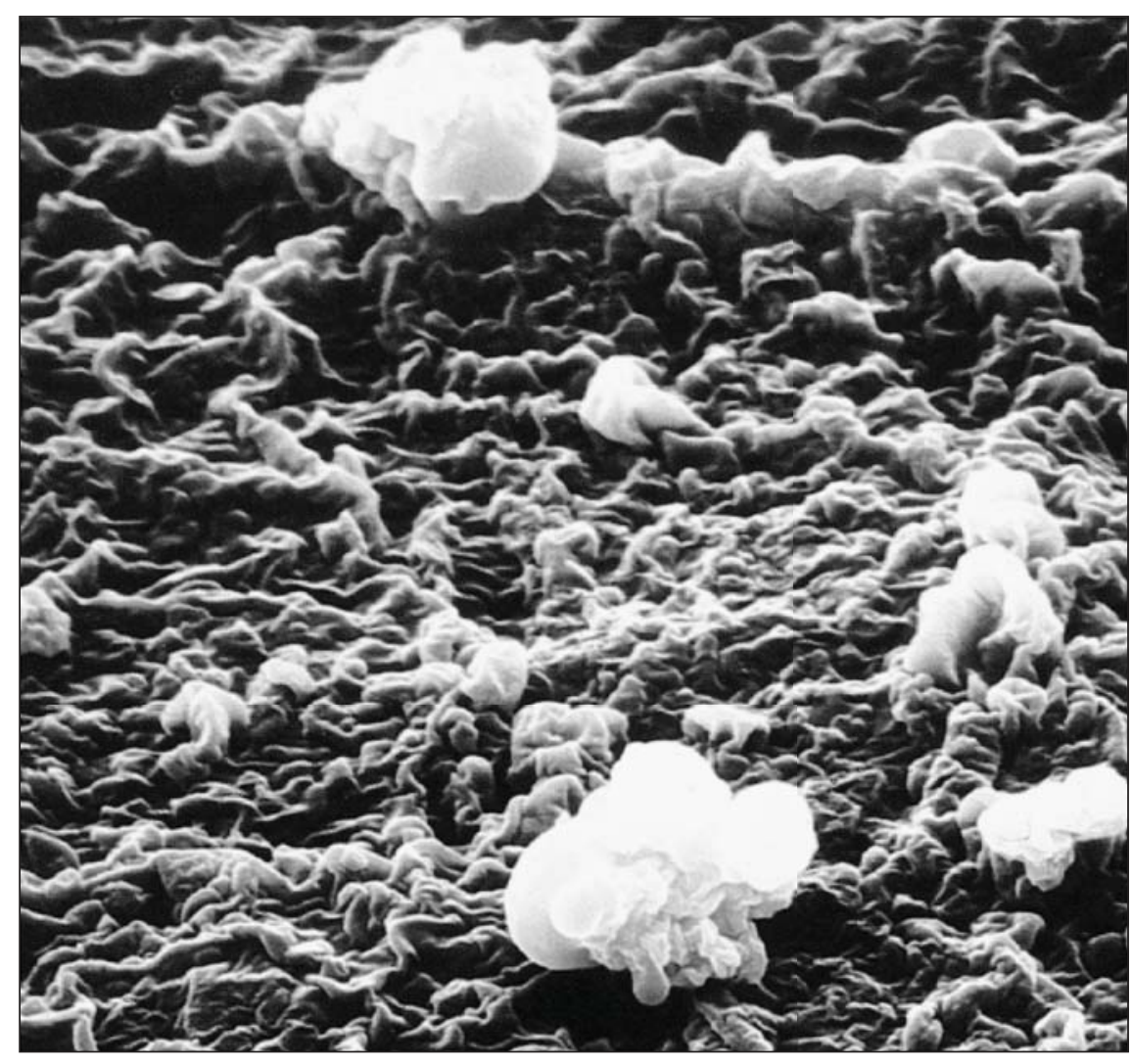

Figure 5. Observation de nanocapsules intactes sous les premières couches cellulaires du stratum cornéum (MEB).

bilité (tableau 3). Cela permet de garantir leur efficacité.

\section{Conclusion}

Les nanocapsules constituent un formidable vecteur pour les molécules cosmétiquement actives car elles garantissent une encapsulation stable et performante en termes de taux de pénétration dans les structures cutanées et de stabilisation. Le procédé adapté à l'encapsulation de molécules «fragiles » a été développé industriellement et nous permet l'utilisation de ce type de capsules à une très large échelle. Cependant, d'autres challenges restent à relever, comme l'augmentation du taux final d'actif encapsulé.

\section{Les nanoémulsions}

Les nanoémulsions [14-16] sont des émulsions très fines, dont le diamètre moyen est inférieur à 100 nm. Contrairement aux microémulsions, les nanoémulsions sont des systèmes métastables, dont la structure dépend de leur procédé de fabrication. Elles peuvent être préparées selon la méthode PIT [17], par émulsification spontanée [18] avec des équipements à fort cisaillement $[14,15]$. giles par nature. Comme elles sont transparentes et généralement très fluides, le moindre petit signe de déstabilisation est visible. Le produit devient opaque et un phénomène de crémage est observé. Nous avons identifié deux sources principales d'instabilité : I'une est liée à la nature de la phase grasse et la seconde à la présence d'un polymère introduit comme agent épaississant ou gélifiant dans la nanoémulsion.

La très faible taille de ces systèmes ( $D$ $50 \mathrm{~nm}$ ) leur confère des propriétés caractéristiques facilement valorisables en cosmétique. Elles
Les nanoémulsions sont des systèmes très fra-

Tableau 3. Stabilité de différentes molécules encapsulées dans les nanocapsules ou dans une émulsion de même taille.

\begin{tabular}{|c|c|c|}
\hline Molécule encapsulée & $\begin{array}{l}\text { Stabilité en nanocapsule } \\
\text { après } 2 \text { mois à } 45^{\circ} \mathrm{C}(\%)\end{array}$ & $\begin{array}{l}\text { Stabilité en émulsion } \\
\text { après } 2 \text { mois à } 45^{\circ} \mathrm{C}(\%)\end{array}$ \\
\hline Rétinol & -6 & -60 \\
\hline B Carotène & 0 & -40 \\
\hline Lycopène & -10 & -60 \\
\hline
\end{tabular}

sont transparentes et facilement absorbées par la peau. Elles peuvent être stérilisées par filtration. Elles peuvent conduire à une large gamme de produits allant de formules très fluides jusqu'à des gels parfaitement transparents.

\section{Matériels et méthodes}

Les nanoémulsions sont stabilisées par un mélange de deux tensio-actifs: PEG-8 isostéarate/disodium stéaroyle glutamate (9/1). Le rapport massique de tensio-actif par rapport à la phase grasse est égal à 0,25 . La phase aqueuse contient $18,75 \%$ de glycols.

Différentes huiles cosmétiques de natures chimiques variées (triglycérides, esters, alcanes, silicones) et de différentes masses molaires sont formulées: caprylic/capric triglycéride (h1), huile d'avocat (h2), huile d'abricot (h3), myristate d'isopropyle (h4), palmitate d'isopropyle (h5), néopentanoate d'isostéaryle (h6), palmitate d'octyle (h7), stéarate d'isocétyle (h8), isododécane (h9), C11-13 isoparaffine (h10), isohexadécane (h11), polyisobutylène hydrogéné (h12), huile minérale (h13), et cyclopentasiloxane (h14). La proportion massique d'huile dans l'émulsion est égale à $25 \%$ au maximum.

Afin d'augmenter la viscosité des nanoémulsions, différents polymères sont introduits, comme par exemple un Carbomer et un polymère associatif de type HEUR.

Les tension-actifs sont dispersés dans I'huile ou le mélange huileux. La phase aqueuse est introduite dans la phase grasse et le mélange est homogénéisé à l'aide d'un mixeur. L'émulsion ainsi obtenue est alors affinée à l'aide d'un homogénéisateur à haute pression (1 200 bars).

La taille des gouttes est évaluée par diffusion de la lumière quasi-élastique (B190 Brookhaven Instrument).

\section{Influence de la nature de I'huile sur la stabilité de la nanoémulsion}

Dans une première partie, les nanoémulsions sont formulées à l'aide d'une seule huile, à la teneur de $15 \%$. La plupart des huiles utilisées permettent l'obtention de nanoémulsions transparentes. La turbidité initiale est comprise entre 100 et 600 NTU. Cependant, certaines d'entre elles deviennent opaques plus ou moins rapidement avec le temps.

Dans le cas des systèmes instables, nous avons mis en évidence une augmentation régulière du diamètre des gouttes avec le temps. Le volume des gouttes en fonction du temps (figure 6) suit une loi linéaire $\left(r^{3} \sim t\right)$ caractéristique du phénomène de mûrissement d'Ostwald [19]. La solubilité de l'huile dans la phase aqueuse est telle que les molécules 


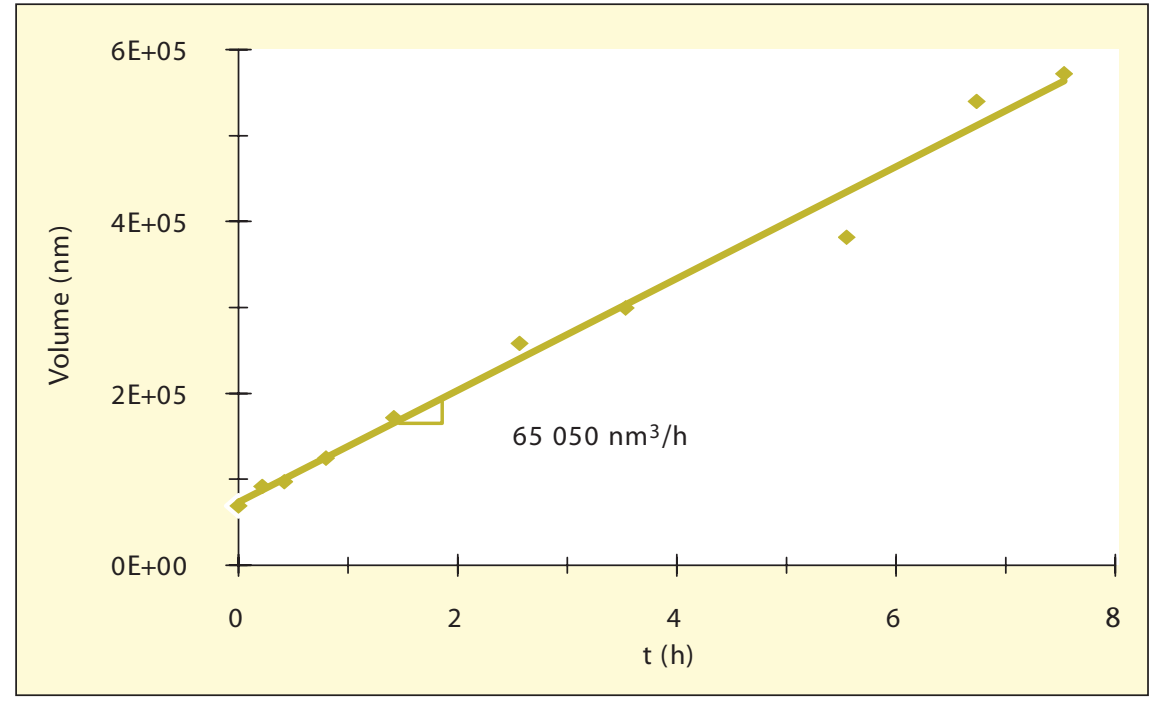

Figure 6. Variation du diamètre et du volume des gouttes en fonction du temps pour l'isopropyl palmitate $\left(T=25^{\circ} \mathrm{C}\right)$. $d^{\prime}$ huile peuvent migrer des petites gouttes vers les plus grosses; la taille moyenne des gouttes augmente ainsi avec le temps.

La figure 6 montre l'évolution du taux de mûrissement $(v)$, qui varie principalement avec la solubilité de l'huile dans l'eau (équation 1):

$$
V=\frac{32}{27} \pi \frac{\gamma V^{m} D s}{R T}\left(\mathrm{~m}^{3} / \mathrm{s}\right)
$$

$\mathrm{s}$ : solubilité de huile dans la phase aqueuse $\left(\mathrm{m}^{3} / \mathrm{m}^{3}\right)$

$\mathrm{D}$ : coefficient de diffusion de l'huile dans la phase aqueuse $\left(\mathrm{m}^{2} / \mathrm{s}\right)$

$\gamma$ : tension interfaciale $(\mathrm{mN} / \mathrm{m})$

$\mathrm{V}^{\mathrm{m}}$ : volume molaire de I'huile $\left(\mathrm{m}^{3} / \mathrm{mol}\right)$

Pour les systèmes instables, nous avons reporté le taux de mûrissement en fonction de l'huile, pour chaque famille, en ordonnant les huiles

par masse molaire croissante (figure 7). Plus I'huile est légère, plus le mûrissement est important.

Pour chaque type d'huile, un système stable peut être obtenu au-delà d'une masse molaire critique. Plus l'huile est polaire, plus la valeur de cette masse molaire critique est importante.

Nous appellerons ainsi une huile "insoluble» (respectivement huile «soluble»), une huile conduisant à une nanoémulsion stable (resp. instable).

\section{Comment concilier stabilité et bonnes propriétés cosmétiques?}

Le phénomène de mûrissement d'Ostwald impose des limitations dans le choix des huiles utilisables. Cependant, la phase grasse des pro-

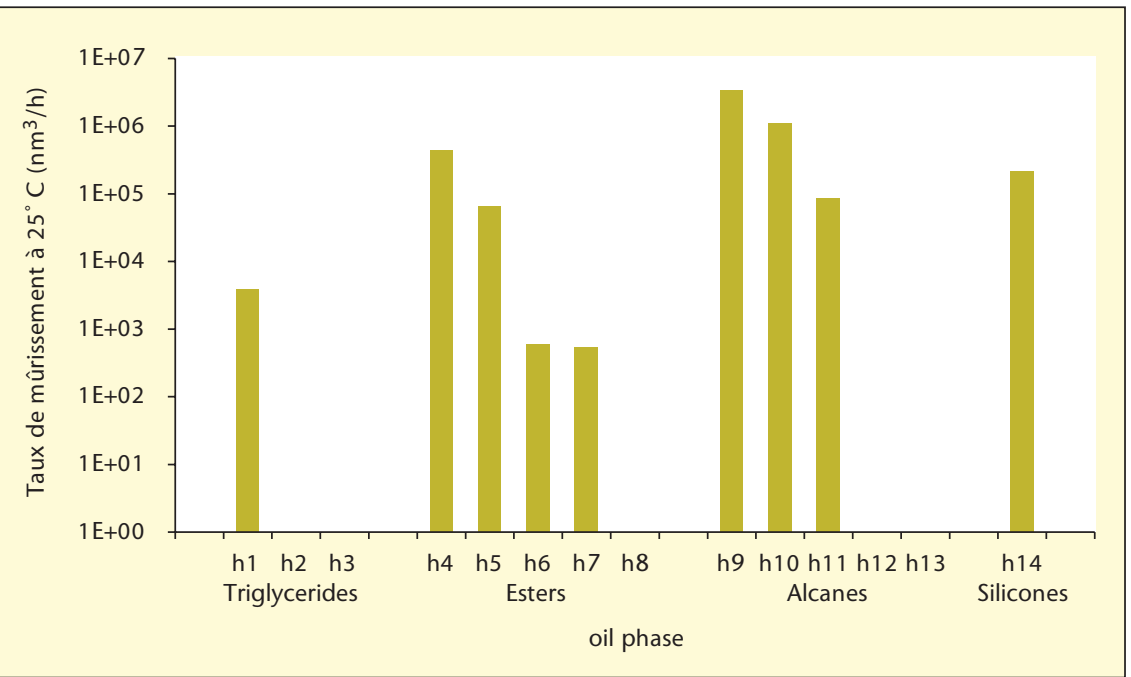

Figure 7. Taux de mûrissement à $25^{\circ} \mathrm{C}$ en fonction de la nature de I'huile. duits cosmétiques est en général composée d'un mélange d'huiles afin d'optimiser les propriétés cosmétiques. Le challenge consiste à savoir comment les choisir et comment les mélanger.

II est possible de mélanger des huiles qui conduisent chacune à une nanoémulsion stable, comme par exemple le polybutylène hydrogéné, l'huile d'abricot et/ou l'isostéarate d'isocétyle. Cependant, ce mélange conduit à un film gras.

La théorie du mûrissement d'Ostwald prédit que les systèmes stables peuvent être obtenus en mélangeant une huile «soluble » avec une huile « insoluble » dans certaines proportions. La fraction molaire de l'huile « insoluble » dans la phase grasse totale doit être supérieure à $x^{*}$, selon l'équation 2 suivante :

$$
x^{2^{*}}=\frac{2 \gamma V_{1}^{m}}{3 R T r}
$$

$\gamma$ : tension interfaciale $(\mathrm{mN} / \mathrm{m})$

$\mathrm{V}^{\mathrm{m}}$ : volume molaire de l'huile $\left(\mathrm{m}^{3} / \mathrm{mol}\right)$

$r$ : diamètre des gouttes $(m)$

Nous avons étudié la stabilité de nanoémulsions comprenant des mélanges d'huiles « insolubles », comme l'isostéarate d'isocétyle, et d'huile "solubles », comme l'isododécane, à différentes proportions massiques (figure 8). Les nanoémulsions restent transparentes pendant deux mois à $45^{\circ} \mathrm{C}$, lorsque que le mélange huileux comprend au moins $50 \%$ en poids d'huile « insoluble».

\section{Nanoémulsions gélifiées ou épaissies par des polymères}

Des polymères ont été utilisés pour moduler la texture des nanoémulsions. Les différents comportements suivants sont observés lors de l'introduction des polymères :

- En présence de carbomers, un blanchiment apparaît quelles que soient la concentration en polymère et la taille des gouttes. Des observations microscopiques montrent la présence d'objets de forme irrégulière et de taille micronique (figure 9), leur nombre étant corrélé à la teneur en polymère. Les petites gouttes $d^{\prime}$ huile

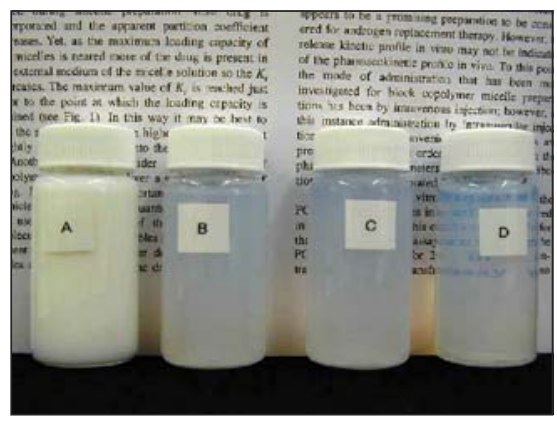

Figure 8. Rapport huile soluble/huile insoluble $=100 / 0$ (A), 70/30 (B), 50/50 (C), 25/75 (D). 


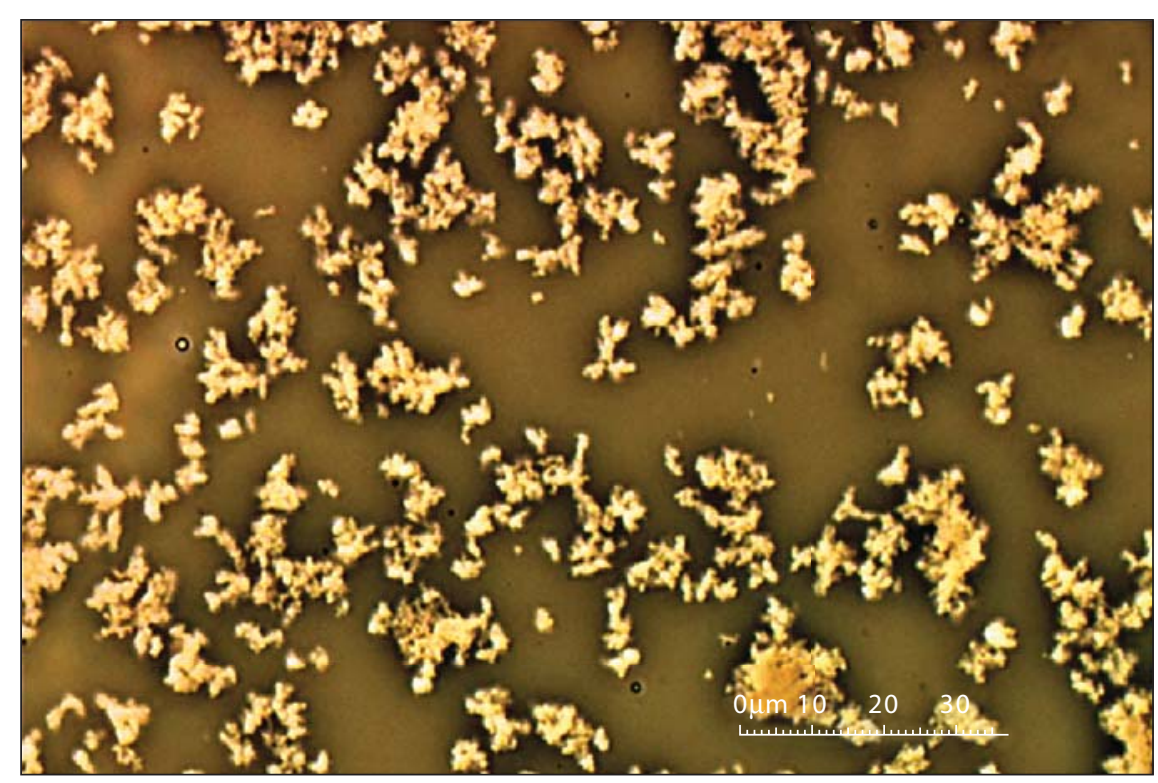

Figure 9. Aspect microscopique d'un mélange d'une nanoémulsion (15\% de phase grasse) et d'un carbomer $(0,2 \%), p H 25^{\prime \prime} /=25^{\prime \prime} / 7$

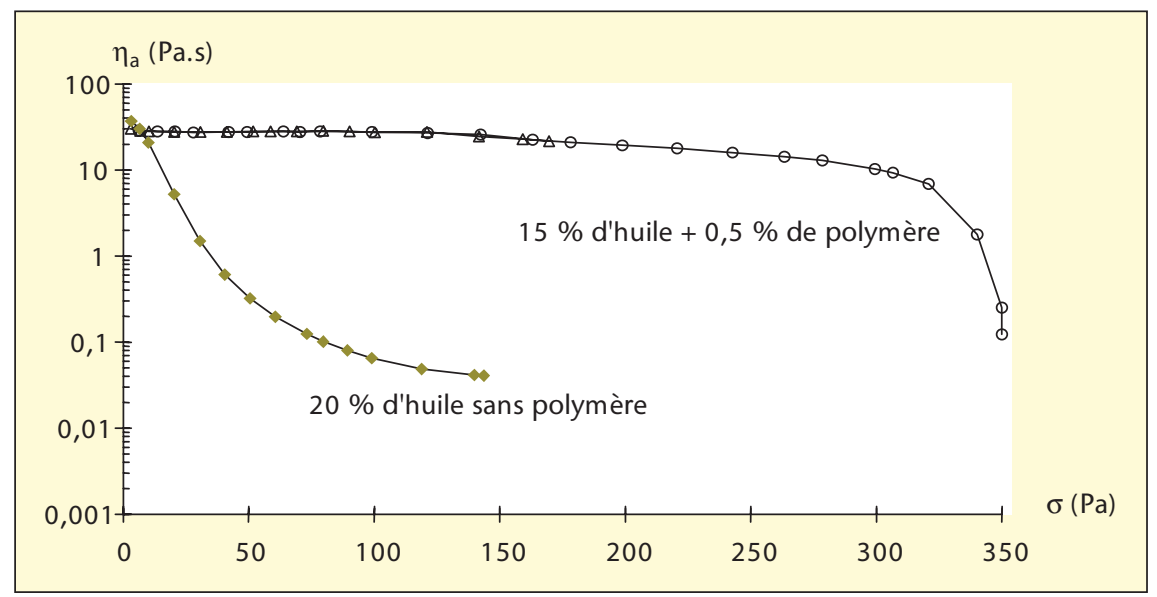

Figure 10. Courbe d'écoulement d'une nanoémulsion comprenant une forte teneur en huile (20\%), en comparaison à celle d'une nanoémulsion (15\% d'huile) épaissie à l'aide d'un polymère associatif.

sont susceptibles de provoquer l'agrégation des macromolécules selon un phénomène de déplétion $[20,21]$.

- Les meilleurs résultats sont obtenus en présence de polymères associatifs de type HEUR, qui conduisent à des nanoémulsions transparentes et épaissies, et présentant de bonnes qualités cosmétiques. Les gouttes participent à la formation d'un réseau tridimensionnel formé par le polymère dans l'eau. Le gel ainsi formé présente des propriétés d'écoulement très différentes de celles des nanoémulsions riches en huile (> $20 \%$ ), d'aspect également gélifié (figure 10).

\section{Propriétés cosmétiques des nanoémulsions}

Les nanoémulsions sont facilement valorisables dans les produits de soin de la peau en raison de leurs propriétés biophysiques et sensorielles spécifiques. Le pouvoir hydratant d'une nanoémulsion a été mesuré à l'aide d'un cornéomètre, une heure après traitement de la peau. Une augmentation significative de l'hydratation est observée pour la nanoémulsion, en comparaison avec la macroémulsion correspondante. de la macroémulsion correspondante a été
Le taux de pénétration d'une nanoémulsion et déterminé à l'aide de mesures du coefficient de frottement de la peau pendant I'application des produits. Les données obtenues montrent que la nanoémulsion pénètre plus rapidement que la macroémulsion correspondante.

\section{Conclusion}

En raison des applications potentielles des nanoémulsions en cosmétique, une étude large a été réalisée afin de déterminer le rôle exact de chacun des constituants. Pour chaque système, les mécanismes de déstabilisation tels que le mûrissement d'Ostwald et la floculation ont été analysés ; la formulation et la stabilité de ces nouvelles dispersions liquides/liquides sont maintenant précisément contrôlées.

\section{RÉFÉRENCES}

1. TIARSK, et al. Langmuir $2001 ; 17: 908-18$.

2. MILLER. Colloïds \& Surfaces $1988 ; 29: 89-102$.

3. SHAHIDZADEH, et al. Europhysics latters 1997 ; 40(4) : 459-64.

4. TORZA, et al. / Colloid Interface Sci $1970 ; 33$ : 6783.

5. AL KHOURY, et al. Int J Pharm $1986 ; 28: 125$ 32.

6. GALLARDO, et al. Int / Pharm $1993 ; 100: 55$.

7. EP 274961 (CNRS).

8. EP447318 (L'ORÉAL).

9. WO 93/05753 (L'ORÉAL).

10. FR 2790405 (L'ORÉAL).

11. FR 2787729 (L'ORÉAL).

12. FR2787730 (L'ORÉAL).

13. FR2742677 (L'ORÉAL).

14. NAKAJIMA H, TOMOMASA S, OKABE M. In : Proceedings of the first World Congress on Emulsions, Paris. $1963: 11-162 ; 1$.

15. ОКАMOTO T, TOMOMASA S, NAKAIIMA H, KAKOKI H, UCHIBORI H, SAITO T. Proceedings of the IFSCC, Venise. 1994.

16. EP-A-728 460

17. FÖRSTER T, VON RYBINSKI W, WADLE A. Adv Colloid Interface Sci 1995 ; 58 : 119-49.

18. EL-AASSER MS, LACK CD, MIN TI, VANDERHOFF JW, FOWKES FM. Colloids ans Surfaces $1984 ; 62$ : 79-97.

19. KABALNOV AS, PERTZOV AV, SCHCHUKIN ED. J Colloid Interface Sci 1987 ; 118 : 590-7.

20. SPERRY PR, HOPFENBERG HB, THOMAS NL. I Colloid Interface Sci $1981 ; 82(1)$ : 62.

21. SPERRY PR. / Colloid Interface Sci $1982 ; 87(2)$ : 375. 